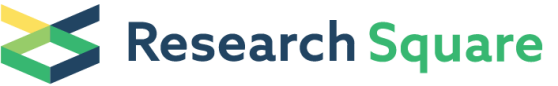 \\ Preprints are preliminary reports that have not undergone peer review. \\ They should not be considered conclusive, used to inform clinical practice, or referenced by the media as validated information.
}

\section{A Nomogram for Predicting In-Hospital Mortality in Patients with Takotsubo Syndrome}

\section{Jun Chen}

The Third Affiliated Hospital of Zhejiang Chinese Medical University

\section{Yimin Wang}

The Third Affiliated Hospital of Zhejiang Chinese Medical University

\section{Xinyang Shou}

The Third Affiliated Hospital of Zhejiang Chinese Medical University

Qiang Liu

The Third Affiliated Hospital of Zhejiang Chinese Medical University

Ziwei Mei ( $\sim$ lszxyymzw@163.com)

Lishui Central Hospital, the Fifth Affiliated Hospital of Wenzhou Medical College

\section{Research Article}

Keywords: Takotsubo syndrome, prognosis, in-hospital mortality, nomogram, MIMIC-IV database.

Posted Date: December 2nd, 2021

DOI: https://doi.org/10.21203/rs.3.rs-1100517/v1

License: (c) (1) This work is licensed under a Creative Commons Attribution 4.0 International License. Read Full License 


\section{Abstract \\ BACKGROUND}

Despite the large number of studies focus on the prognosis and in-hospital outcomes risk factors of patients with takotsubo syndrome, there was still lack of utility and visual risk prediction model for predicting the in-hospital mortality of patients with takotsubo syndrome.

\section{OBJECTIVES}

Our study aimed to establish a utility risk prediction model for the prognosis of in-hospital patients with takotsubo syndrome (TTS).

\section{METHODS}

The study is a retrospective cohort study. Model of in-hospital mortality of TTS patients was developed by multivariable logistic regression analysis. Calibration and discrimination were used to assess the performance of the nomogram. The clinical utility of the model was evaluated by decision curve analysis (DCA).

\section{RESULTS}

Overall, 368 TTS patients (320 Survivals and 48 deaths) were included in our research from MIMIC-IV database. The incidence of in-hospital mortality with TTS is $13.04 \%$. Lasso regression and multivariate logistic regression model verified that potassium, pt, age, myocardial infarction, WBC, hematocrit, anion gap and SOFA score were significantly associated with in-hospital mortality of TTS patients. The nomogram demonstrated a good discrimination with a AUC of ROC $0.811(95 \% \mathrm{Cl}$ : $0.746-0.876)$ in training set and $0.793(95 \% \mathrm{Cl}: 0.724-0.862)$ in test set. The calibration plot of risk prediction model showed predicted probabilities against observed death rates indicated excellent concordance. DCA showed that the nomogram has good clinical benefits.

\section{Conclusion}

We developed a nomogram that predict hospital mortality in patients with TTS according to clinical data. The nomogram exhibited excellent discrimination and calibration capacity, favoring its clinical utility.

\section{Introduction}


Takotsubo syndrome (TTS) is a clinical syndrome also viewed as "stress cardiomyopathy," "broken heart syndrome," or "transient apical ballooning" and is characterized by an acute and transient left ventricular dysfunction which always induced by physical or emotional stimulation ${ }^{1}$. Takotsubo syndrome is easily misdiagnosed as acute coronary syndrome (ACS), approximately $1 \%-3 \%$ of all patients who present with symptoms consistent with acute coronary syndrome and undergo coronary angiography have been identified to TTS $^{2}$. Despite numerous studies reported that TTS was a reversible myocardial insufficiency and TTS patients always have a good prognosis during the hospitalization ${ }^{3-6}$. However, several serious complications may increase mortality to a rate, which is comparable with that of patients with $\mathrm{ACS}^{7}$. In the recent studies, the mortality rates have been reported higher than the previous studies ${ }^{8}$. Recently, one research based on the data from the SCAAR (Swedish Coronary Angiography and Angioplasty Registry) reported that the 30-day mortality rate in TTS was lower than ST-segment-elevation myocardial infarction (STEMI) but higher than non-STEMI (NSTEMI) ${ }^{9}$. Previous study on in-hospital outcomes of TTS patients discussed around risk factors abount mortality. Nevertheless, the predictive model of in-hospital mortality of TTS patients stay in ICU was not quite established. Therefore, assessment of the risk of in-hospital mortality in TTS patients by clinical predictive model can effectively provide a reference for subsequent hospitalization treatment and nursing. These measures would play an important role in improving the prognosis of patients with TTS.

At present, clinical prediction model is a good tool and can provide valuable guidance for clinical decision making. Nomogram as a special visual clinical prediction model that can calculates the risk scores for individuals, is convenient for clinicians to use in prognosis prediction. It has been widely used as a predictive method in the evaluation of prognosis in critically ill patients in recent years ${ }^{10-14}$. However, there was limited nomogram for predicting the hospitalization prognosis of patients with TTS. Therefore, we aimed to develop a nomogram for predicting the risk of in-hospital mortality in patients with TTS to identify the high risk of death patients early. This work would provide adequate reference for subsequent decision making, treatment and intensive care.

\section{Methods}

\section{Data Source}

This was a retrospective observational study, the primary data of our study was derived from MIMIC-IV database (version 1.0). MIMIC-IV database is an extensive database, and contained all medical record numbers corresponding to patients admitted to an intensive care unit (ICU) or the emergency department between 2008-2019 in the Beth Israel Deaconess Medical Center (BIDMC) ${ }^{15}$. The version 1.0 is the lasted version of MIMIC-IV database. One of our authors (C.J, certification ID: 8979131) gained permission to documented the database after online training at the National Institutes of Health (NIH). All methods were carried out in accordance with relevant guidelines to protect the privacy of patients.

\section{Patients selection}


We enrolled patients who aged more than 18 years old stay in ICU and diagnosed with TTS by the International Classification of Diseases version 9 ("42983") and version 10 diagnosis codes ("I5181") in the MIMIC-IV database. We excluded patients according to the following criteria: (I) No survival outcome data; (II) Being pregnancy and the postpartum condition; (III) Incomplete or unobtainable documented or other vital medical data records.

\section{Clinical and laboratory data}

Extracted the basic information including age, height, weight and medical history such as diabetes, hypertension, chronic lung disease, myocardial infarction, heart failure, et al of TTS patients. Extracted vital signs information and the blood laboratory tests information of TTS patients as the first document after patients admitted to the hospital within 24 hours. Vital signs data contained mean blood pressure (MBP), diastolic blood pressure (DBP), systolic blood pressure (SBP), body temperature (T), respiratory rate (RR), heart rate (HR), pulse oximetry derived oxygen saturation (spo2). Blood laboratory tests data consisted of hemoglobin, hematocrit, creatinine, anion gap, lactate, blood urea nitrogen (BUN), $\mathrm{PH}$, white blood cell count, platelet count, chloride, glucose, prothrombin time (PT), serum potassium, serum sodium, and serum calcium. Therapies were also recorded, which contained the use of vasoactive drugs (norepinephrine), and continuous renal replacement therapy (CRRT) during the hospitalization. The sequential organ failure assessment (SOFA) score $^{16}$ of every patient also should be calculated. In our study, the endpoint was in-hospital mortality viewed as survival status at hospital discharge.

\section{Statistical Analysis}

The whole dataset was randomly divided into a training set and a test set with the proportion of 7:3. Mean \pm standard deviation (SD) documented normal distribution of continuous variables. Medians with upper and lower quartiles described unnormal distribution of continuous variables. Continuous variables were tested by T-test or Wilcoxon rank-sum test and categorical variables were analysed by chi-square test or Fisher's exact test for group comparisons. First, lasso regression was used to conduct preliminary screening of the predictors based on the whole study database, and screened out the predictors with large regression coefficients. Second, multivariate regression analysis was applyed to analyze the above screened predictors and established the nomogram in training dataset. The scores for each predictors were calculated based on coefficients of logistic regression variables in the model. We adopted receiver operating characteristic curve (ROC) to assess the discrimination of nomogram for in-hospital mortality of TTS patients. The area under the curve (AUC) of the ROC curve more than 0.7 was defined as good discrimination. The degree of fitting of the nomogram was assessed by calibration curve analysis which tested by Hosmer-Lemeshow test. The decision curve analysis (DCA) was conducted to evaluate the clinical utility of the nomogram through quantifying net benefits against a range of threshold probabilities. The validation of nomogram capabilities were used by test set. These results were presented by odds ratio (OR) with $95 \%$ confidence intervals (Cls). All tests were two-tailed tests and $p \leq$ 0.05 was considered statistically significant. We used STATA 15.0 (Stata Corporation, College Station, Texas, USA) and R version 3.6.3 (R Foundation for Statistical Computing, Vienna, Austria) for statistical analysis. 


\section{Results}

\section{The characteristics of study patients}

Totally, we included 368 eligible patients (85 males and 283 females) in our study, their average age was $66.29 \pm 16.09$ years old, more information about missing data and data extraction process was shown in Supplementary Table S1 and Table S2. 48 patients (16 males and 32 females) died during the hospitalization, with the incidence of in-hospital death rate was $13.04 \%$. Death group patients tend to older, and with higher value of anion gap, WBC count and SOFA score (As showed in Table1). The whole sample was randomly divided into a training dataset and a test dataset with the proportion of 7:3, and there were no significant difference in observed clinical variables between the training set and the validation set (As showed in Table 2). 
Table 1

Baseline characteristics of study patients stratified by survival outcome

\begin{tabular}{|c|c|c|c|c|}
\hline Characteristic & Total $(n=368)$ & Survival $(n=320)$ & death $(n=48)$ & $P$ value \\
\hline Age (years old) & $66.29 \pm 16.09$ & $65.42 \pm 16.31$ & $72.09 \pm 13.30$ & 0.007 \\
\hline Man & $85(23.10 \%)$ & $69(21.56 \%)$ & $16(33.33 \%)$ & 0.105 \\
\hline $\mathrm{SBP}(\mathrm{mmHg})$ & $112.00 \pm 15.25$ & $112.43 \pm 15.40$ & $109.60 \pm 18.36$ & 0.170 \\
\hline $\mathrm{DBP}(\mathrm{mmHg})$ & $65.70 \pm 10.64$ & $62.72 \pm 10.86$ & $60.91 \pm 12.60$ & 0.913 \\
\hline $\mathrm{MBP}(\mathrm{mmHg})$ & $78.65 \pm 10.43$ & $78.88 \pm 10.69$ & $74.80 \pm 12.85$ & 0.284 \\
\hline Heart rate (beats/minute) & $91.40 \pm 17.08$ & $90.40 \pm 16.71$ & $89.48 \pm 19.66$ & 0.147 \\
\hline Respiratory rate (beats/minute) & $21.03 \pm 4.12$ & $20.98 \pm 4.11$ & $22.06 \pm 4.63$ & 0.549 \\
\hline Temperature $\left({ }^{\circ} \mathrm{C}\right)$ & $36.89 \pm 0.48$ & $36.89 \pm 0.48$ & $36.27 \pm 1.21$ & 0.518 \\
\hline SPO2 (\%) & $96.95 \pm 2.35$ & $96.89 \pm 2.43$ & $94.95 \pm 7.49$ & 0.231 \\
\hline \multicolumn{5}{|l|}{ Comorbidities, n (\%) } \\
\hline Diabetes & $89(24.18 \%)$ & $77(24.06 \%)$ & $12(25.00 \%)$ & 1.000 \\
\hline Hypertension & $157(42.66 \%)$ & $141(44.06 \%)$ & $16(33.33 \%)$ & 0.213 \\
\hline myocardial infarction & $111(30.16 \%)$ & $102(31.88 \%)$ & $9(18.75 \%)$ & 0.093 \\
\hline congestive heart failure & $212(57.61 \%)$ & $187(58.44 \%)$ & $25(52.08 \%)$ & 0.500 \\
\hline Chronic pulmonary disease & $99(26.90 \%)$ & $82(25.63 \%)$ & $17(35.42 \%)$ & 0.211 \\
\hline Malignant cancer & $55(14.95 \%)$ & $47(14.69 \%)$ & $8(16.67 \%)$ & 0.887 \\
\hline Renal disease & $58(15.76 \%)$ & $51(15.94 \%)$ & $7(14.58 \%)$ & 0.978 \\
\hline \multicolumn{5}{|l|}{ Laboratory parameters } \\
\hline anion gap (mEq/L) & $17.63 \pm 5.41$ & $15.64 \pm 4.02$ & $17.53 \pm 4.69$ & 0.003 \\
\hline $\mathrm{BUN}(\mathrm{mg} / \mathrm{dL})$ & $33.83 \pm 24.64$ & $23.84 \pm 18.59$ & $26.35 \pm 13.70$ & 0.369 \\
\hline Bicarbonate (mmol/L) & $20.80 \pm 5.18$ & $22.11 \pm 4.52$ & $20.83 \pm 5.18$ & 0.075 \\
\hline Creatinine (mg/dL) & $1.96 \pm 1.98$ & $1.24 \pm 1.35$ & $1.16 \pm 0.72$ & 0.697 \\
\hline Chloride (mmol/L) & $103.83 \pm 6.66$ & $103.64 \pm 6.48$ & $101.78 \pm 7.77$ & 0.072 \\
\hline Glucose $(\mathrm{mg} / \mathrm{dL})$ & $188.89 \pm 94.74$ & $171.85 \pm 70.62$ & $203.25 \pm 109.05$ & 0.706 \\
\hline
\end{tabular}

SBP: systolic blood pressure; DBP: diastolic blood pressure; MBP: mean blood pressure; SPO2: pulse oximetry derived oxygen saturation; BUN: blood urea nitrogen; INR: international nominal ratio; WBC: white blood cell; SOFA: sequential organ failure assessment; APSII: acute physiology score II; ICU: intensive care unit; HOS: hospital; LOS: length of stay. 


\begin{tabular}{|c|c|c|c|c|}
\hline Characteristic & Total $(n=368)$ & Survival $(n=320)$ & death $(n=48)$ & $P$ value \\
\hline calcium & $8.36 \pm 1.19$ & $8.22 \pm 0.80$ & $8.06 \pm 0.89$ & 0.198 \\
\hline Hematocrit (\%) & $32.70 \pm 6.37$ & $33.03 \pm 6.29$ & $30.54 \pm 6.49$ & 0.011 \\
\hline Hemoglobin $(\mathrm{g} / \mathrm{dL})$ & $10.69 \pm 2.15$ & $10.79 \pm 2.13$ & $9.98 \pm 2.19$ & 0.015 \\
\hline WBC $\left(10^{9} / \mathrm{L}\right)$ & $13.83 \pm 7.74$ & $13.46 \pm 7.72$ & $16.27 \pm 7.45$ & 0.019 \\
\hline Platelet $\left(10^{9} / \mathrm{L}\right)$ & $240.37 \pm 139.90$ & $239.79 \pm 138.51$ & $244.25 \pm 150.35$ & 0.837 \\
\hline Potassium (mmol/L) & $4.20 \pm 0.63$ & $4.17 \pm 0.63$ & $4.35 \pm 0.61$ & 0.067 \\
\hline PT & $15.91 \pm 8.13$ & $15.41 \pm 6.94$ & $19.22 \pm 13.25$ & 0.002 \\
\hline Sodium (mmol/L) & $138.19 \pm 5.25$ & $138.31 \pm 5.03$ & $137.40 \pm 6.52$ & 0.259 \\
\hline Renal replacement therapy,n (\%) & $18(4.89 \%)$ & $15(4.69 \%)$ & $3(6.25 \%)$ & 0.913 \\
\hline norepinephrine, $\mathrm{n}(\%)$ & 147 (39.95\%) & $126(39.38 \%)$ & $21(43.75 \%)$ & 0.675 \\
\hline \multicolumn{5}{|l|}{ Scoring systems } \\
\hline SOFA & $5.98 \pm 4.00$ & $5.78 \pm 3.87$ & $7.27 \pm 4.64$ & 0.016 \\
\hline ICU LOS, days & $5.82 \pm 6.75$ & $5.85 \pm 9.19$ & $5.56 \pm 6.17$ & 0.782 \\
\hline HOS LOS (days) & $17.75 \pm 17.91$ & $18.51 \pm 18.34$ & $14.20 \pm 14.30$ & 0.120 \\
\hline \multicolumn{5}{|c|}{$\begin{array}{l}\text { SBP: systolic blood pressure; DBP: diastolic blood pressure; MBP: mean blood pressure; SPO2: pulse } \\
\text { oximetry derived oxygen saturation; BUN: blood urea nitrogen; INR: international nominal ratio; WBC: } \\
\text { white blood cell; SOFA: sequential organ failure assessment; APSII: acute physiology score Il; ICU: } \\
\text { intensive care unit; HOS: hospital; LOS: length of stay. }\end{array}$} \\
\hline
\end{tabular}


Table 2

The characteristic of training dataset and test dataset subjects

\begin{tabular}{|c|c|c|c|c|}
\hline Characteristic & Total $(n=368)$ & $\begin{array}{l}\text { Training set } \\
(\mathrm{n}=258)\end{array}$ & $\begin{array}{l}\text { Test set } \\
(n=110)\end{array}$ & $\begin{array}{l}P \\
\text { value }\end{array}$ \\
\hline Age (years old) & $66.29 \pm 16.09$ & $66.29 \pm 16.31$ & $66.27 \pm 15.63$ & 0.988 \\
\hline Man & $85(23.10 \%)$ & $55(21.31 \%)$ & $30(27.30 \%)$ & 0.269 \\
\hline $\mathrm{SBP}(\mathrm{mmHg})$ & $112.00 \pm 15.25$ & $111.72 \pm 15.35$ & $112.67 \pm 15.07$ & 0.583 \\
\hline $\mathrm{DBP}(\mathrm{mmHg})$ & $65.70 \pm 10.64$ & $65.75 \pm 10.60$ & $65.56 \pm 10.80$ & 0.874 \\
\hline $\mathrm{MBP}(\mathrm{mmHg})$ & $78.65 \pm 10.43$ & $78.38 \pm 10.39$ & $79.29 \pm 10.53$ & 0.448 \\
\hline Heart rate (beats/minute) & $91.40 \pm 17.08$ & $91.94 \pm 16.59$ & $90.12 \pm 18.20$ & 0.348 \\
\hline $\begin{array}{l}\text { Respiratory rate } \\
\text { (beats/minute) }\end{array}$ & $21.03 \pm 4.12$ & $21.10 \pm 4.24$ & $20.87 \pm 3.83$ & 0.627 \\
\hline Temperature $\left({ }^{\circ} \mathrm{C}\right)$ & $36.89 \pm 0.48$ & $36.88 \pm 0.50$ & $36.93 \pm 0.41$ & 0.314 \\
\hline SPO2 (\%) & $96.95 \pm 2.35$ & $96.91 \pm 2.14$ & $97.04 \pm 2.78$ & 0.645 \\
\hline \multicolumn{5}{|l|}{ Comorbidities, n (\%) } \\
\hline Diabetes & $89(24.18 \%)$ & $58(22.48 \%)$ & $31(28.19 \%)$ & 0.300 \\
\hline Hypertension & $157(42.66 \%)$ & $112(43.41 \%)$ & $45(40.91 \%)$ & 0.410 \\
\hline myocardial infarction & $111(30.16 \%)$ & $68(26.36 \%)$ & $43(39.10 \%)$ & 0.021 \\
\hline congestive heart failure & $212(57.61 \%)$ & $147(56.98 \%)$ & $65(59.10 \%)$ & 0.794 \\
\hline Chronic pulmonary disease & $99(26.90 \%)$ & $73(28.29 \%)$ & $26(23.64 \%)$ & 0.427 \\
\hline Malignant cancer & $55(14.95 \%)$ & $41(15.89 \%)$ & $14(12.73 \%)$ & 0.603 \\
\hline Renal disease & $58(15.76 \%)$ & $46(17.83 \%)$ & $12(10.91 \%)$ & 0.131 \\
\hline \multicolumn{5}{|l|}{ Laboratory parameters } \\
\hline anion gap (mEq/L) & $17.63 \pm 5.41$ & $15.75 \pm 4.05$ & $16.20 \pm 4.40$ & 0.342 \\
\hline $\mathrm{BUN}(\mathrm{mg} / \mathrm{dL})$ & $33.83 \pm 24.64$ & $24.47 \pm 18.81$ & $23.47 \pm 16.12$ & 0.630 \\
\hline Bicarbonate (mmol/L) & $20.80 \pm 5.18$ & $21.99 \pm 4.43$ & $21.84 \pm 5.07$ & 0.776 \\
\hline Creatinine $(\mathrm{mg} / \mathrm{dL})$ & $1.96 \pm 1.98$ & $1.24 \pm 1.17$ & $1.20 \pm 1.51$ & 0.799 \\
\hline Chloride (mmol/L) & $103.83 \pm 6.66$ & $103.29 \pm 6.68$ & $103.64 \pm 6.70$ & 0.652 \\
\hline
\end{tabular}

SBP: systolic blood pressure; DBP: diastolic blood pressure; MBP: mean blood pressure; SPO2: pulse oximetry derived oxygen saturation; BUN: blood urea nitrogen; INR: international nominal ratio; WBC: white blood cell; SOFA: sequential organ failure assessment; APSII: acute physiology score II; ICU: intensive care unit; HOS: hospital; LOS: length of stay. 


\begin{tabular}{|c|c|c|c|c|}
\hline Characteristic & Total $(n=368)$ & $\begin{array}{l}\text { Training set } \\
(n=258)\end{array}$ & $\begin{array}{l}\text { Test set } \\
(n=110)\end{array}$ & $\begin{array}{l}\mathrm{P} \\
\text { value }\end{array}$ \\
\hline Glucose (mg/dL) & $188.89 \pm 94.74$ & $149.41 \pm 53.65$ & $203.25 \pm 109.05$ & $<0.001$ \\
\hline calcium & $8.36 \pm 1.19$ & $8.18 \pm 0.83$ & $8.26 \pm 0.78$ & 0.358 \\
\hline Hematocrit (\%) & $32.70 \pm 6.37$ & $33.33 \pm 6.27$ & $33.58 \pm 6.52$ & 0.084 \\
\hline Hemoglobin $(\mathrm{g} / \mathrm{dL})$ & $10.69 \pm 2.15$ & $10.58 \pm 2.14$ & $10.92 \pm 2.16$ & 0.159 \\
\hline WBC $\left(10^{9} / \mathrm{L}\right)$ & $13.83 \pm 7.74$ & $13.87 \pm 7.49$ & $13.73 \pm 8.33$ & 0.871 \\
\hline Platelet $\left(10^{9} / \mathrm{L}\right)$ & $240.37 \pm 139.90$ & $243.91 \pm 144.80$ & $232.05 \pm 127.92$ & 0.457 \\
\hline Potassium (mmol/L) & $4.20 \pm 0.63$ & $4.19 \pm 0.65$ & $4.21 \pm 0.59$ & 0.801 \\
\hline PT & $15.91 \pm 8.13$ & $15.56 \pm 6.72$ & $16.72 \pm 10.71$ & 0.210 \\
\hline Sodium (mmol/L) & $138.19 \pm 5.25$ & $138.00 \pm 5.43$ & $138.64 \pm 4.80$ & 0.287 \\
\hline $\begin{array}{l}\text { Renal replacement therapy, } \mathrm{n} \\
(\%)\end{array}$ & $18(4.89 \%)$ & $14(5.43 \%)$ & $4(3.64 \%)$ & 0.642 \\
\hline norepinephrine, n (\%) & 147 (39.95\%) & $104(40.31 \%)$ & $43(39.10 \%)$ & 0.918 \\
\hline \multicolumn{5}{|l|}{ Scoring systems } \\
\hline SOFA & $5.98 \pm 4.00$ & $6.07 \pm 4.07$ & $5.78 \pm 3.86$ & 0.521 \\
\hline ICU LOS, days & $5.82 \pm 6.75$ & $5.85 \pm 6.78$ & $5.80 \pm 6.83$ & 0.944 \\
\hline HOS LOS (days) & $17.75 \pm 17.91$ & $17.68 \pm 17.96$ & $17.68 \pm 18.09$ & 0.885 \\
\hline \multicolumn{5}{|c|}{$\begin{array}{l}\text { SBP: systolic blood pressure; DBP: diastolic blood pressure; MBP: mean blood pressure; SPO2: pulse } \\
\text { oximetry derived oxygen saturation; BUN: blood urea nitrogen; INR: international nominal ratio; WBC: } \\
\text { white blood cell; SOFA: sequential organ failure assessment; APSIl: acute physiology score II; ICU: } \\
\text { intensive care unit; HOS: hospital; LOS: length of stay. }\end{array}$} \\
\hline
\end{tabular}

\section{Predict factors for TTS prognosis}

We performed lasso regression analysis to make a preliminary selection of TTS prognosis predictors. (As showed in Figure 1). Nine predictors were identified having large regression coefficient (large regression coefficient means great influence on the outcome events) which included age, myocardial infarction history, gender, potassium, PT, WBC, hematocrit, anion gap, SOFA score. Multivariable regression analysis demonstrated that age (OR: 1.003; 95\% Cl: 1.001-1.005), myocardial infarction history (OR: 0.920; 95\% Cl: 0.864-0.991), hematocrit (OR: 0.988; 95\% Cl: 0.993-0.999), WBC (OR: 1.004; 95\% Cl: 1.000-1.009), anion gap (OR: 1.011; 95\% Cl: 1.002-1.019), PT (OR: 1.005; 95\% Cl: 1.001-1.009), SOFA score (OR: 1.010; 95\% Cl: 1.001-1.017) were the independent risk factors for in-hospital mortality of TTS patients. However, there was no statistical difference in gender and serum potassium concentration. Considering the importance of gender and serum potassium concentration in TTS patients, our model included them in the nomogram finally. 


\section{Nomogram for predicting the risk of in-hospital mortality in patients with TTS}

The nomogram included age, myocardial infarction history, gender, potassium, pt, WBC, hematocrit, anion gap, SOFA score as predictive factors for in-hospital mortality of TTS patients. For example, a 74-yearsold male without myocardial infarction history, his clinical data of admission as followed: PT: 30 seconds, potassium: $5 \mathrm{mmol} / \mathrm{L}$, anion gap: $24 \mathrm{mEq} / \mathrm{L}$, WBC: $10^{\star} 10^{\wedge} 9$, hematocrit: $35 \%$ and SOFA score was 4 points. The corresponding score of each predictors were 6.5 points, 1.5 points, 2.8 points, 0.6 points, 0.7 points, 6.5 points, 1.5 points, 6.7 points, and 1.2 points respectively. Then his total score was about 28 points, and the risk of in-hospital mortality is 65\%. (As showed in Figure 2).

\section{Performance evaluation and validation of the nomogram}

ROC curve analysis for the training set showed that our risk prediction model has a good discrimination (AUC of ROC: $0.811,95 \% \mathrm{Cl}: 0.746-0.876$, as showed in Figure 3(a)). We validated the prediction model in the test set, and the result showed that the present prediction model also has a good discrimination in test set (AUC of ROC: $0.793,95 \% \mathrm{Cl}$ : 0.724-0.862, as showed in Figure 3(b)). Furthermore, we performed calibration curve analysis to test the fitting degree of nomogram. The calibration curve plot showed that predicted probabilities against observed death rates indicated good concordance (as showed in Figure 3(c)). In the test set, the calibration curve plot also showed good concordance between the predicted probabilities and the observed mortality (as showed in Figure 3(d)). In addition, we evaluate the clinical utility of the prediction model by decision curve analysis. DCA curve showed that our prediction model has a utility clinical practice (as showed in Figure 3(e) and Figure 3(f)).

\section{Discussion}

In the retrospective observational study, we found that the TTS patients have a hospital mortality rate of $13.04 \%$. This rate of in-hospital mortality in our study was higher than the rate $2-5 \%$ in previous studies ${ }^{3-}$ 6 , this was due to our study only included the TTS patients admitted to ICU, these patients tend to have more serious and complicated illness condition than the TTS patients in previous studies reported. Our study demonstrated that age, myocardial infarction history, PT, WBC, hematocrit, anion gap, SOFA score were important predictive factors for in-hospital mortality of severe TTS patients. Despite gender (male) and serum potassium concentration in our study without statistical difference (as shown in Table 3), several previous studies have identified that gender (male) have a higher in-hospital mortality compared with female ${ }^{17-18}$, thus, we also included gender as a predictor in the nomogram. In addition, multiple studies have reported that serum potassium concentration was associated with poor prognosis of acute heart failure patients ${ }^{19-20}$. Considering that almost all the TTS patients have moderate to severe cardiac insufficiency, thus, we included serum potassium in the final nomogram. The nomogram based on above predictors showed a good predictive ability. 
Table 3

Lasso and multivariable regression analysis of risk factors

\begin{tabular}{|lllll|}
\hline \multirow{2}{*}{ variable } & \multicolumn{2}{l}{ Lasso regression analysis } & \multicolumn{2}{l|}{ Multivariable regression analysis } \\
\cline { 2 - 5 } & OR $(95 \%$ Cl) & P value & OR $(95 \%$ Cl) & P value \\
\hline Age (years old) & $1.003(1.001-1.005)$ & 0.024 & $1.003(1.001-1.005)$ & 0.016 \\
\hline Gender (man) & $1.078(0.994-1.170)$ & 0.072 & $1.051(0.970-1.139)$ & 0.104 \\
\hline myocardial infarction & $0.924(0.870-0.988)$ & 0.041 & $0.920(0.864-0.991)$ & 0.043 \\
\hline Hematocrit & $0.991(0.985-0.999)$ & 0.009 & $0.988(0.993-0.999)$ & 0.011 \\
\hline WBC & $1.008(1.003-1.014)$ & 0.005 & $1.004(1.000-1.009)$ & 0.032 \\
\hline anion gap & $1.014(1.004-1.024)$ & 0.008 & $1.011(1.002-1.019)$ & 0.026 \\
\hline Potassium & $1.039(0.975-1.108)$ & 0.238 & $1.025(0.971-1.083)$ & 0.876 \\
\hline PT & $1.003(0.997-1.009)$ & 0.121 & $1.005(1.001-1.009)$ & 0.045 \\
\hline SOFA & $1.011(1.001-1.021)$ & 0.038 & $1.010(1.001-1.017)$ & 0.047 \\
\hline WBC: white blood cell; PT: prothrombin time; SOFA: sequential organ failure assessment \\
\hline
\end{tabular}

Gender is closely related to the onset and prognosis of TTS patients. Male with TTS were reported to have a higher in-hospital mortality compared with female $17,18,21$ and were viewed as an independent risk factor for in-hospital death in TTS patients in previous study ${ }^{22}$. In addition, male were related to adverse composite events consist of cardiovascular death, severe pump failure, and fatal ventricular arrhythmias (odds ratio $4.32,95 \% \mathrm{Cl} 1.41-13.6$ ) reported by a multicenter registry study of $\mathrm{TTS}^{23}$. The reason for male gender is more likely to be in-hospital death probably is that men are more possible to exit comorbidities such as COPD, coronary artery disease, a higher peak troponin-l level and to burden the severity of myocardial injury than women. Moreover, men were more likely to have a physical stressor compared with women, TTS patients induced by physicology were more likely to have malignancy, lower hemoglobin, higher serum creatinine and died in the hospital than unphysicology 24 .

For women, their organism release estrogen which could mitigates myocardial damage. The protective action for myocardial injury performed by estrogen was demonstrated by several studies in animal experimental models ${ }^{25}$. In our study, $23.1 \%$ of our TTS patients were male and the in-hospital death rate was $37.65 \%(32 / 85)$ in males. On the contrary, the in-hospital death rate was $5.65 \%(16 / 283)$ in females. It seems that males have higher in-hospital mortality compared with female $(37.65 \%$ vs $5.65 \%, P<0.001)$. This result was same as the study performed by Yoshihiro Sobue et $\mathrm{al}^{22}$. However, to our surprise, gender was not found to be a risk factor for in-hospital death in either univariate or multivariate regression analyses. Recently, Budnik $\mathrm{M}$ et al study found that inhospital outcomes (arrhythmias, mechanical complications, cardiogenic shock, mortality rate) were similar in both male and female groups after 
adjusted other confounding factors ${ }^{26}$. These results suggested that gender contribute for the in-hospital death of TTS should be further research.

The sequential organ failure assessment (SOFA) score was applied to describe the time course of multiple organ dysfunction. Recent studies showed that SOFA score was associated with survival in severe cases ${ }^{27}$. Previous study revealed that increase SOFA during the first few days of ICU admission is a good indicator of prognosis. Both the mean and highest SOFA scores are particularly useful predictors of outcome ${ }^{28}$. In our study, the SOFA score of in-hospital death patients was statistically higher than survival patients $(7.27$ vs $5.78, P=0.016)$. Multivariate logistic regression analysis demonstrates that sofa score was an independent predictive factor for in-hospital mortality in TTS patients. Therefore, SOFA score could be a good predictor for prognosis of TTS patients in intensive care units.

We included myocardial infarction history as predictor in the nomogram and found for the first time patients with myocardial infarction history have lower in-hospital death rate than others without myocardial infarction history. This point hasn't been reported in previous study. Less is known on the inhospital outcome role of myocardial infarction history in TTS. In our study, survival patients with myocardial infarction was statistically higher than in-hospital death patients $(31.88 \%$ vs $18.75 \%$, $\mathrm{P}=0.093)$. The protective role of myocardial infarction history on in-hospital outcome for TTS patients deserved us to investigate in the following research.

Currently, there were limited effective clinical predictive model to predict the in-hospital mortality in TTS patients. To the best of our knowledge, it's the first nomogram for predicting the in-hospital mortality of TTS patients. Santoro F et al. developed the GEIST prognosis score (male sex, history of neurologic disorder, right ventricular involvement, and left ventricular ejection fraction (LVEF)) for risk stratification for in-hospital complications in patients with $\mathrm{TTS}^{29}$. Compared with Santoro F et al. study, our study focused on the vital signs and blood laboratory tests information which were more easier to obtain. We performed lasso regression and logistic regression to analysis the relevant clinical indicators in the hospital outcomes of TTS patients, pick out the most important predictors. The results of performance evaluation and validation revealed that our nomogram has a good discrimination. Echocardiographic parameters can provide additional information to identify higher risk of hemodynamic deterioration patients compared to other routine clinical information ${ }^{30}$. Citro $\mathrm{R}$ et al. revealed that LVEF, E/e' ratio, reversible moderate to severe mitral regurgitation were independent correlates of major adverse events ${ }^{30}$. These parameters have important clinical value, and future studies can add cardiac hyperparameters on the basis of our study to increase the prediction efficiency of the prediction model.

\section{Study limitation}

Several limitations must be acknowledged. First, we aimed to establish a rapid and simple nomogram for predicting in-hospital mortality in TTS patients. The situation of TTS patients was very complex and there were many factors related to the prognosis of their hospitalization. We only included the clinical data of the patients within 24 hours after admission, and did not consider the intervention measures at the onset 
or after hospitalization, which has certain limitations; Second, our study did not further analyze the causes of TTS, such as physical triggers or psychological triggers. Future studies can conduct a subgroup analysis of specific causes of cardiac arrest for provide more evidence for this term.

\section{Conclusions}

We have established a risk prediction model by using admission characteristics of TTS patients could help identify in-hospital mortality in TTS patients.

\section{Abbreviations}

TTS: takotsubo syndrome; ICD: International Classification of Diseases; AUC: area under the curve; ROC: receiver operating characteristic; Cl: confidence intervals; SBP: systolic blood pressure; DBP: diastolic blood pressure; MBP: mean blood pressure; SPO2: pulse oximetry derived oxygen saturation; BUN: blood urea nitrogen; PT: prothrombin time; WBC: white blood cell; CRRT: continuous renal replacement therapy; SOFA: sequential organ failure assessment; ICU: intensive care unit; HOS: hospital; LOS: length of stay.

\section{Declarations}

Acknowledgements $\square$ We acknowledge MIMIC database for providing their platforms and contributors for uploading their meaningful datasets.

Competing interests: All other authors have no conflict of interest.

Authors' contributions: LQ and MZW conceived and designed research; CJ, WYM and MZW collected data and conducted research; CJ, SXY and WYM analyzed and interpreted data; CJ wrote the initial paper; MZW revised the paper; CJ had primary responsibility for final content. All authors read and approved the final manuscript.

Funding: Zhejiang traditional Chinese medicine science and technology plan project(2019ZQ047), Wu Jieping Medical Foundation(320.6750.2020-04-44)

Availability of data and materials: The datasets generated and analyzed during the current study are available from the corresponding author on reasonable request.

Ethics approval and consent to participate: The establishment of this database was approved by the Massachusetts Institute of Technology (Cambridge, MA) and Beth Israel Deaconess Medical Center (Boston, MA), and consent was obtained for the original data collection. Therefore, the ethical approval statement and the need for informed consent were waived for this manuscript.

Consent for publication: Not applicable. 


\section{References}

1. Ali M, Rigopoulos AG, Ali K, Ikonomidis I, Makavos G, Matiakis M, Melnyk H, Abate E,Mammadov M, Prüser JL, de Vecchis R, Wohlgemuth W, Manginas A, Bigalke B, Mavrogeni S, Sedding D, Noutsias M. Advancements in the diagnostic workup, prognostic evaluation, and treatment of takotsubo syndrome. Heart Fail Rev. 2020 Sep;25(5):757-771. doi: 10.1007/s10741-019-09843-9.

2. Akashi YJ, Nef HM, Lyon AR. Epidemiology and pathophysiology of Takotsubo syndrome. Nat Rev Cardiol. 2015 Jul;12(7):387-97. doi: 10.1038/nrcardio.2015.39.

3. Cacciotti L, Passaseo I, Marazzi G, Camastra G, Campolongo G, Beni S, Lupparelli F, Ansalone G. Observational study on Takotsubo-like cardiomyopathy: clinical features, diagnosis, prognosis and follow-up. BMJ Open. 2012 Oct 11;2(5):e001165. doi: 10.1136/bmjopen-2012-001165.

4. Dias A, Franco E, Mercedes A, Hebert K, Messina D, Quevedo HC. Clinical features of takotsubo cardiomyopathy - a single-center experience. Cardiology. 2013;126(2):126-30. doi:

10.1159/000353369. Epub 2013 Aug 17. PMID: 23969858.

5. Weihs V, Szücs D, Fellner B, Eber B, Weihs W, Lambert T, Metzler B, Titscher G, Hochmayer B, Dechant C, Eder V, Siostrzonek P, Leisch F, Pichler M, Pachinger O, Gaul G, Weber H, Podczeck-Schweighofer A, Nesser HJ, Huber K. Stress-induced cardiomyopathy (Tako-Tsubosyndrome) in Austria. Eur Heart J Acute Cardiovasc Care. 2013 Jun;2(2):137-46. doi: 10.1177/2048872613483592.

6. Núñez Gil IJ, Andrés M, Almendro Delia M, Sionis A, Martín A, Bastante T, Córdoba Soriano JG, Linares Vicente JA, González Sucarrats S, Sánchez-Grande Flecha A; RETAKO investigators. Characterization of Tako-tsubo Cardiomyopathy in Spain: Results from the RETAKO National Registry. Rev Esp Cardiol (Engl Ed). 2015 Jun;68(6):505-12. doi: 10.1016/j.rec.2014.07.026.

7. Templin C, Ghadri JR, Diekmann J, et al. Clinical Features and Outcomes of Takotsubo (Stress) Cardiomyopathy. N Engl J Med. 2015 Sep 3;373(10):929-38. doi: 10.1056/NEJMoa140676.

8. T. Stiermaier, C. Moeller, K. Oehler, et al., Long-term excess mortality in takotsubo cardiomyopathy: predictors, causes and clinical consequences, Eur. J. Heart Fail. 18(2016) 650-656.

9. Redfors B, Jha S, Thorleifsson S, Jernberg T, Angerås O, Frobert O, Petursson P, Tornvall P,Sarno G, Ekenbäck C, Ravn-Fisher A, Y-Hassan S, Lyon AR, James S, Erlinge D, Omerovic E. Short- and LongTerm Clinical Outcomes for Patients With Takotsubo Syndrome and PatiensWith Myocardial Infarction: A Report From the Swedish Coronary Angiography and Angioplasty Registry. J Am Heart Assoc. 2021 Sep 7;10(17):e017290. doi: 10.1161/JAHA.119.01729.

10. Bai ZH, Guo XQ, Dong R, Lei N, Pei HH, Wang H. A Nomogram to Predict the 28-day Mortality of Critically III Patients With Acute Kidney Injury and Treated With Continuous Renal Replacement Therapy. Am J Med Sci. 2021 May;361(5):607-615. doi: 10.1016/j.amjms.2020.11.028.

11. Yang S, Su T, Huang L, Feng LH, Liao T. A novel risk-predicted nomogram for sepsis associated-acute kidney injury among critically ill patients. BMC Nephrol. 2021 May 10;22(1):173.doi:

10.1186/s12882-021-02379-x. 
12. Zhou Y, He Y, Yang H, Yu H, Wang T, Chen Z, Yao R, Liang Z. Development and validation a nomogram for predicting the risk of severe COVID-19: A multi-center study in Sichuan,China. PLoS One. 2020 May 18;15(5):e0233328. doi: 10.1371/journal.pone.0233328.

13. Liu J, Tao L, Gao Z, Jiang R, Liu M. Development and validation of a prediction model for early identification of critically ill elderly COVID-19 patients. Aging (Albany NY). 2020 Oct 6;12(19):1882218832. doi: 10.18632 /aging. 103716.

14. Jiang X, Su Z, Wang Y, Deng Y, Zhao W, Jiang K, Sun C. Prognostic nomogram for acute pancreatitis patients: An analysis of publicly electronic healthcare records in intensive care unit. J Crit Care. 2019 Apr;50:213-220. doi: 10.1016/j.jcrc.2018.10.030.

15. Johnson, A., Bulgarelli, L., Pollard, T., Horng, S., Celi, L. A., \& Mark, R. (2021). MIMIC-IV (version 1.0). PhysioNet. https://doi.org/10.13026/s6n6-xd98.

16. Raith EP, Udy AA, Bailey M, McGloughlin S, Maclsaac C, Bellomo R, Pilcher DV; Australian and New Zealand Intensive Care Society (ANZICS) Centre for Outcomes and Resource Evaluation (CORE). Prognostic Accuracy of the SOFA Score, SIRS Criteria, and qSOFA Score for In-Hospital Mortality Among Adults With Suspected Infection Admitted to the Intensive Care Unit. JAMA. 2017 Jan 17;317(3):290-300. doi: 10.1001/jama.2016.20328.

17. Lemor A, Ramos-Rodriguez AJ, De La Villa R, Hosseini Dehkordi SH, Vazquez de Lara F,Lee S, Rodriguez Rivera M, Casso Dominguez A, Argulian E. Impact of gender on in-hospitaloutcomes in patients with Takotsubo syndrome: A nationwide analysis from 2006 to 2014. ClinCardiol. 2019 Jan;42(1):13-18. doi: 10.1002/clc.23109.

18. Pérez-Castellanos A, Martínez-Sellés $M$, Mejía-Rentería $H$, Andrés $M$, Sionis A, Almendro-Delia $M$, Martín-García A, Aguilera MC, Pereyra E, Linares Vicente JA, García de la Villa B,Núñez-Gil IJ. Takotsubo Syndrome in Men: Rare, but With Poor Prognosis. Rev Esp Cardiol (Engl Ed). 2018 Sep;71(9):703-708. English, Spanish. doi: 10.1016/j.rec.2017.07.021.

19. Krogager ML, Eggers-Kaas L, Aasbjerg K, Mortensen RN, Køber L, Gislason G, Torp-Pedersen C, Søgaard P. Short-term mortality risk of serum potassium levels in acute heart failure following myocardial infarction. Eur Heart J Cardiovasc Pharmacother. 2015 Oct;1(4):245-51. doi:10.1093/ehjcvp/pvv026.

20. Caravaca Perez P, González-Juanatey JR, Nuche J, Morán Fernández L, Lora Pablos D, AlvarezGarcía J, Bascompte Claret R, Martínez Selles M, Vázquez García R, Martínez Dolz L, Cobo-Marcos M, Pascual Figal D, Crespo-Leiro MG, Nuñez Villota J, Cinca Cuscullola J, Delgado JF. Serum potassium dynamics during acute heart failure hospitalization. Clin Res Cardiol.2020 Oct 17. doi: 10.1007/s00392-020-01753-3.

21. Yerasi C, Tripathi B, Banga S et al. Predictors of 90-Day Readmission and in-Hospital Mortality in Takotsubo Cardiomyopathy: An Analysis of 28,079 Index Admissions. Cardiovasc Revasc Med. 2019 Nov;20(11):973-979. doi: 10.1016/j.carrev.2019.08.004.

22. Sobue $Y$, Watanabe $E$, Ichikawa $T$ et al. Physically triggered Takotsubo cardiomyopathy hasa higher in-hospital mortality rate. Int J Cardiol. 2017 May 15;235:87-93. doi: 10.1016/j.ijcard.2017.02.090. 
Epub 2017 Feb 23. PMID: 28262347.

23. Murakami T, Yoshikawa T, Maekawa Y, Ueda T, Isogai T, Sakata K, Nagao K, YamamotoT, Takayama M. Gender Differences in Patients with Takotsubo Cardiomyopathy: Multi-Center Registry from Tokyo CCU Network. PLoS One. 2015 Aug 28;10(8): e0136655. doi:10.1371/journal.pone.0136655

24. Pelliccia F, Pasceri V, Patti G et al. Long-Term Prognosis and Outcome Predictors in Takotsubo Syndrome: A Systematic Review and Meta-Regression Study. JACC Heart Fail. 2019 Feb;7(2):143154. doi: 10.1016/j.jchf.2018.10.009. Epub 2019 Jan 2. PMID: 30611720.

25. Babiker FA, Joseph S, Juggi J. The protective effects of 17 beta-estradiol against ischemiareperfusion injury and its effect on pacing postconditioning protection to the heart. J Physiol Biochem. 2014 Mar;70(1):151-62. doi: 10.1007/s13105-013-0289-9.

26. Budnik M, Nowak R, Fijałkowski M, Kochanowski J, Nargiełło E, Piątkowski R, Peller M,Kucharz J, Jaguszewski M, Gruchała M, Opolski G. Sex-dependent differences in clinical characteristics and inhospital outcomes in patients with takotsubo syndrome. Pol Arch Intern Med.2020 Jan 31;130(1):2530. doi: 10.20452/pamw.14970.

27. de Grooth HJ, Geenen IL, Girbes AR, Vincent JL, Parienti JJ, Oudemans-van Straaten HM.SOFA and mortality endpoints in randomized controlled trials: a systematic review and meta-regression analysis. Crit Care. 2017 Feb 24;21(1):38. doi: 10.1186/s13054-017-1609-1.

28. Ferreira FL, Bota DP, Bross A, Mélot C, Vincent JL. Serial evaluation of the SOFA score to predict outcome in critically ill patients. JAMA. 2001 Oct 10;286(14):1754-8. doi: 10.1001/jama.286.14.1754.

29. Santoro F, Núñez Gil IJ, Stiermaier T, El-Battrawy I, Guerra F, Novo G, Guastafierro F, Tarantino N, Novo S, Mariano E, Romeo F, Romeo F, Capucci A, Bahlmann E, Zingaro M, Cannone M, Caldarola P, Marchetti MF, Montisci R, Meloni L, Thiele H, Di Biase M, Almendro-Delia M, Sionis A, Akin I, Eitel I, Brunetti ND. Assessment of the German and Italian StressCardiomyopathy Score for Risk Stratification for In-hospital Complications in Patients With Takotsubo Syndrome. JAMA Cardiol. 2019 Sep 1;4(9):892-899. doi: 10.1001/jamacardio.2019.2597.

30. Citro R, Rigo F, D'Andrea A, Ciampi Q, Parodi G, Provenza G, Piccolo R, Mirra M, ZitoC, Giudice R, Patella MM, Antonini-Canterin F, Bossone E, Piscione F, Salerno-Uriarte J; Tako-Tsubo Italian Network Investigators. Echocardiographic correlates of acute heart failure, cardiogenic shock, and in-hospital mortality in tako-tsubo cardiomyopathy. JACC Cardiovasc Imaging.2014 Feb;7(2):119-29. doi: 10.1016/j.jcmg.2013.09.020.

\section{Figures}



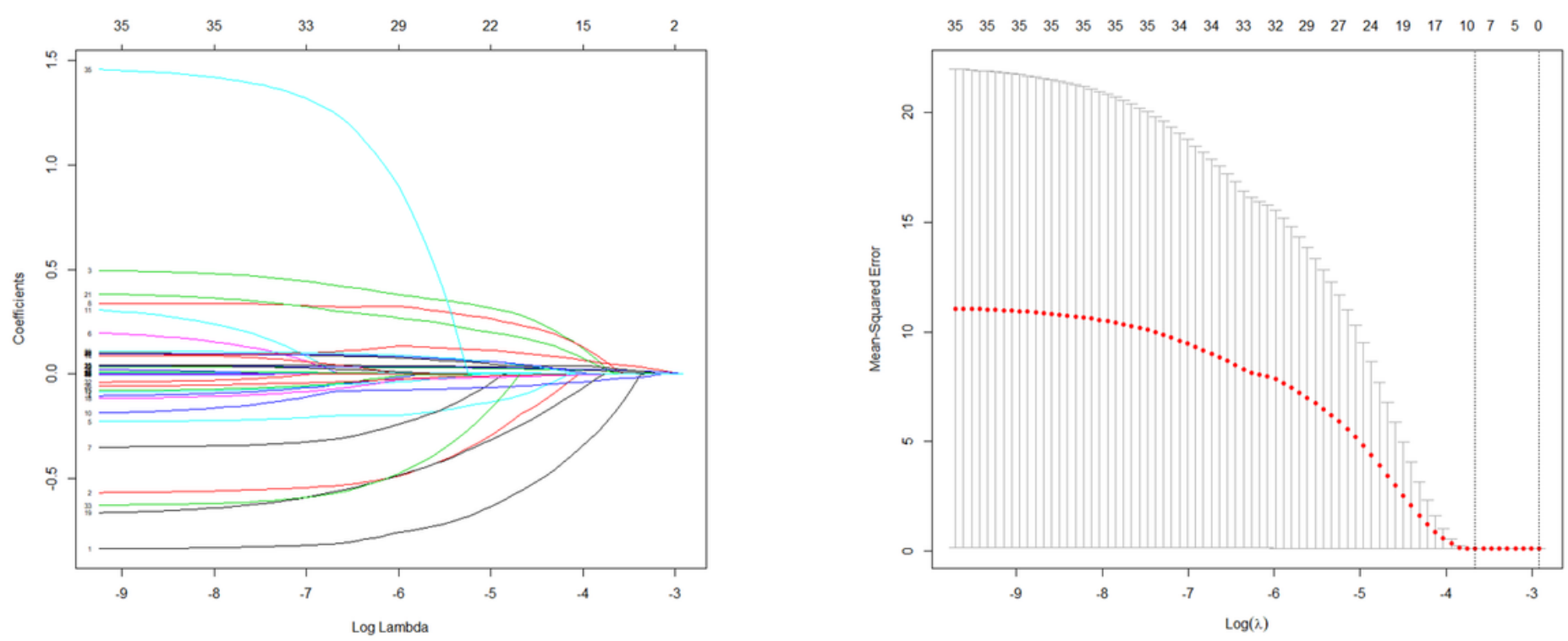

Figure 1

lasso regression analysis; (a) lasso regression analysis; (b) cross validation

\section{Nomogram}

sofa

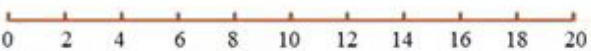

$\mathrm{pt}$

$\begin{array}{llll}9 & 30 & 51 & 73\end{array}$

potassium $\quad 2 \quad 4 \quad 6$

aniongap

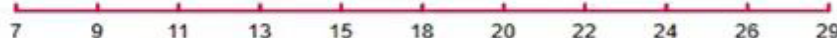

wbc

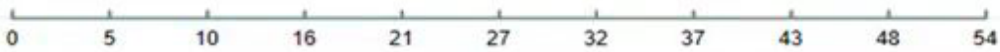

hematocrit

gender
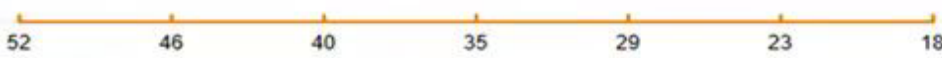

admission_age

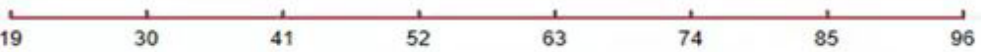

myocardial_infarction

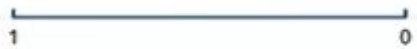

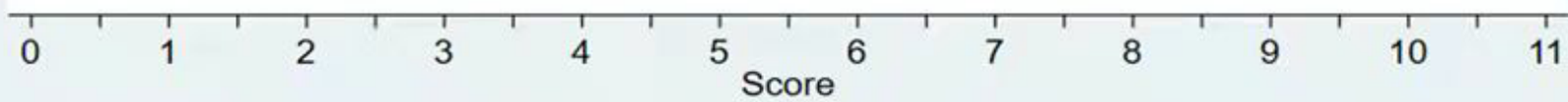

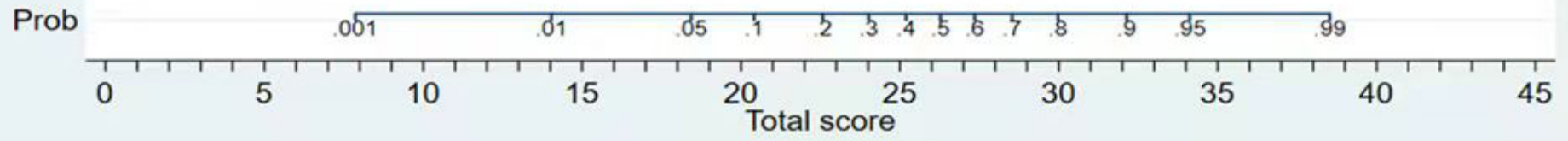

Figure 2

nomogram of in-hospital mortality predictive model in TTS patients 

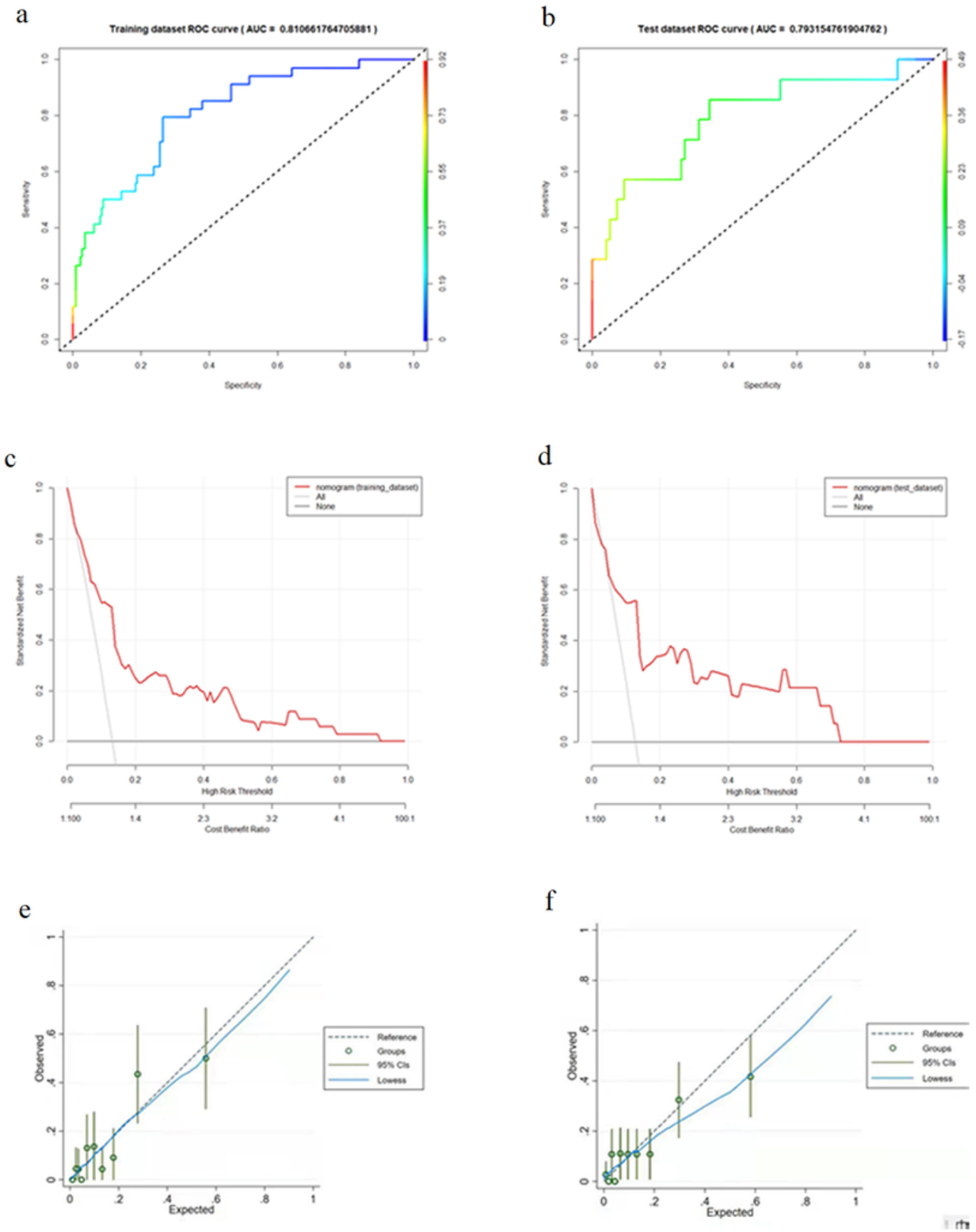

\section{Figure 3}

performance evaluation and validation of the nomogram; (a) ROC curve analysis of the training set; (b) ROC curve analysis of the test set; (c) the calibration curve plot of the training set; (d) the calibration curve plot of the test set; (e) DCA curve of the training set; (f) DCA curve of the test set.

\section{Supplementary Files}


This is a list of supplementary files associated with this preprint. Click to download.

- TableS1andTableS2.docx 